\title{
Editorial: Green Cloud Computing and Communication
}

\section{H. Abdul Shabeer ${ }^{1}$ Palaniappan Palani Ramaswamy ${ }^{2} \cdot$ Hameed Abdul Zubar $^{3}$ - R. S. D. Wahida Banu ${ }^{4}$}

Published online: 2 June 2020

(C) Springer Science+Business Media, LLC, part of Springer Nature 2020

\section{Editorial:}

The worldwide growing energy demands together with the increasing depletion of fossil fuels have been recognized as a major challenge that needs to be urgently addressed by society in order to have a sustainable future. This realization has led to a push towards "Green Cloud computing and Communications" that can enable a transition towards a more sustainable society with a reduced carbon footprint is of prime importance. A major role in the "greening" effort to globally reduce energy consumption will be played by Computing, Communication and Fixed networking technologies. All these technologies have to face the critical issues on energy consumption, for example, in industries like healthcare, largescale cloud datacenters etc. Therefore, new architectures, systems and related application techniques, such as mobile cloud computing (MCC), software-defined networking (SDN), Clustering, Fixed Network Connection and hybrid cloud services, are very much needed in cloud computing datacenters and in communication \& network devices in order to be more productive and energy efficient, for shaping a "Green" world in the future. In this context, there is a critical need to find out a new ways in reducing energy consumption for better environment.

This special issue features six selected papers with high quality. The first article, "A Comparative Study between Iterative Algorithms for TDOA Based Geolocation Techniques in Real UMTS Networks" authored by Juan Anthonio et.al., has compared famous Levenberg-Marquardt

H. Abdul Shabeer

abdulshabeer@gmail.com

1 AVS Engineering College, 2, Military Rd, Ammapet, Salem 636003, India

2 School of Engineering, Faculty of Science and Engineering, University of Wolverhampton, Wulfruna St, Wolverhampton WV1 1LY, UK

3 Department of Mechanical Engineering, Knowledge Institute of Technology, Knowledge College Rd, Kakapalayam 637504, India

4 Anna University, Chennai 600025, India and Gauss-Newton algorithms to solve a multilateration problem with unknown synchronization between the base stations is analyzed. Results shown proved Levenberg-Marquardt method offers better results, providing faster convergence, higher accuracy and more robustness. The author has also made a modification to the classical Levenberg-Marquardt algorithm to avoid divergences with tested results.

The second article titled "Industrie 4.0 Readiness: Green Computing in relation with Key Performance Indicators for a Manufacturing Industry" authors here explores the Indutry 4.0 readiness of an SME under the environmental constraints. They have defined the key performance indicators (KPIs) and use a discrete-event simulation model for a tile manufacturing unit. It describes the environmental constraints as extremely challenging for the small and medium enterprises, to cope with in the realm of Industry 4.0. With limited resources of SMEs, it is sometimes prohibiting to invest in research, simulation analysis and optimization to improve the process. A template-based process is usually adopted. Authors have claim that similar strategies can be used in other SMEs and the improved values for the KPIs pay back in terms of energy-saving measures or energy auditing, thus contributing to the environment.

In the next article with the title "Green Computing Process and its Optimization using Machine Learning Algorithm in Healthcare sector", proposed an optimized algorithm for predicting heart diseases in early stages and at the same time the accuracy of prediction results are also quite convincing. It uses hybridized new machine learning K nearest Neighbor and Spiral Optimization algorithm for implementation. This paper will give a new direction to the Big data analytic researchers in Healthcare Sector.

The fourth article titled, "A DE-ANN Inspired Skin Cancer Detection Approach using Fuzzy C-Means Clustering", authored by Manoj Kumar et.al., proposed a systematic solution for medical imaging in detecting type of skin lesions quickly and accurately. An improved strategy to detect three type of skin cancers in early stages have also suggested. The considered input is a skin lesion image which by using the proposed method, the system would classify it into cancerous or non-cancerous type of skin. Further, the image 
segmentation is implemented by using fuzzy C-means clustering to separate homogeneous image regions in-order to detect the type of skin pathology.

The fifth article, "Healthcare Services Monitoring in Cloud Using Secure and Robust Healthcare-Based Blockchain (SRHB)Approach" authored by Azath Mubarakali, proposed a Health Electronic Records (HER) which share the data to improve the quality and decrease the cost of Healthcare using block chain technology. The proposed method is highly sensitive for securing private data for the determination and transformation of information in the healthcare domain using blockchain. The patient health records are collected from wearable devices with a token-based blockchain approach and shared encrypted data for uploading in the cloud server securely for further analysis and investigation.

The last article titled "Collaborative Detection and Mitigation of Distributed Denial-of-Service Attacks on Software-Defined Network" proposed by Omer and Marsono provides a mechanism to identify the anomaly detection and mitigation system for Distributed Denial-ofService (DDoS) traffic flows, sflow-RT, Snort rules and RSMQ. The proposed method allows the Redis Simple Message Queue (RSMQ) mechanism to update the Ryu controllers with the latest DDoS attack which will prevent any DDoS attack from entering others Software-Defined Network (SDN) sites. Collaborative technique to detect and mitigate DDoS flooding attacks on SDN. This technique integrates sflow-RT application and Snort rules for the detection of DDoS traffic flows in an SDN controller. RSMQ acts as a mechanism to share DDoS detection and mitigation rules among multiple Ryus SDN controllers. The rule-sharing allows a reduction of the controller's overhead for processing DDoS detection and mitigation.

Acknowledgments The guest editors are thankful to our reviewers for their effort in reviewing the manuscripts. We also thank the Edit-inChief, Dr. Imrich Chlamtac for his supportive guidance during the entire process. We also like to extend our sincere gratitude to Eliška Vlčková, Leonora M. Panday and Jed Joseph Adel for their continuous support and regular followup's in successfully completing this special issue.

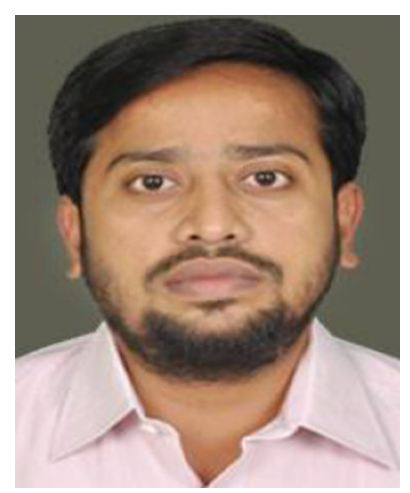

Dr. H. Abdul Shabeer obtained his B.E. in Computer Science and Engineering 2005, Anna University, Chennai, M.E in VLSI Design in the year 2007 and Ph.D in Mobile Computing in the year 2012 from Anna University, Chennai. He has registered 2 Patents and has published more than 60 International Journal and Conferences. He has also served as a Lead Guest Editor in various journals like Springer, Elsevier, Inderscience, Taylor \& Francis, CSIR etc., He worked as team lead in Bank of New Work, Mellon and also worked as a mainframe Lead Specialist in IBM India Pvt. Ltd., Bangalore, India. Currently, he is working as a Dean- Research in AVS Engineering College, Salem, India. He was Member and senior member in Various Professional Societies like IEEE, ISTE, IETE etc. His area of interest includes Mobile Communication, Image and Video Processing, Neural Networks, AI, Transport and Safety, VLSI, Circuit and Devices.

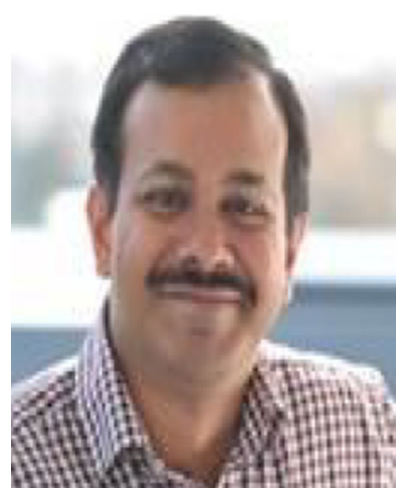

Dr Palaniappan ('Palani') Ramaswamy received his first degree and M.Eng.Sc. degree in Electrical engineering and his $\mathrm{Ph}$. D. degree in Microelectronics/ Telecommunication engineering in 1997, 1999, and 2002, respectively. He is currently a Reader in Signal Analysis in the School of Engineering, Faculty of Science and Engineering, University of Wolverhampton, UK. His research interests include Brain Computer Interface, Networking, Switching, neural-networks, genetic-algorithms, $\mathrm{AI}$ and image processing. To date, he has written two text books in engineering and published over 180 papers (with citation count over 1400 ) in peer-reviewed journals, book chapters, and conference proceedings. He is a senior member of the Institute of Electrical and Electronics Engineers, member in Institution of Engineering and Technology. He also serves as editorial board member for several international journals and serves in national and international grant funding bodies. 


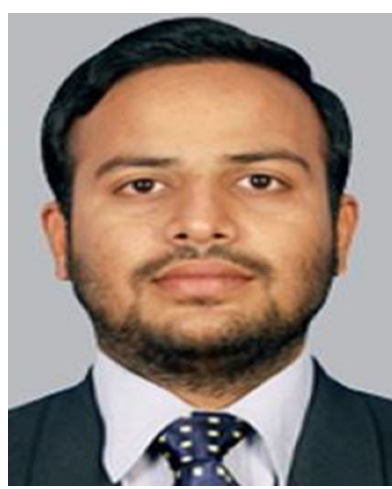

Dr. Hameed Abdul Zubar is an Associate professor in department of Mechanical Engineering at Knowledge Institute of Technology, Salem, India. He completed his Post Graduate and Ph.D. at National Institute of Technology, Tiruchirappalli, Tamil Nadu, India. He has 12 years of teaching experience and 4 years of industrial experience. He specialized in Performance Measurement in Health Care Service, Safety measurement in Supply Chain Management, Occupational Health and Safety Management. He presented and published 36 international conferences in various recognized institutions and also, he published 37 papers in international journals. He is acting as a reviewer and guest editor of reputed journals worldwide.

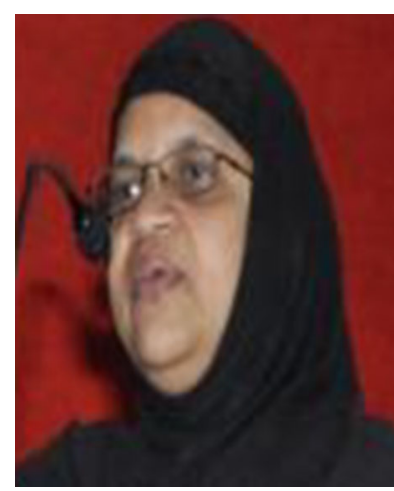

Dr. R. S. D. Wahida Banu obtained her B.E. degree in 1981 and M.E. degree in 1984 from Madras University and Ph.D in 1998 from Anna University, Chennai. She is the First lady Doctorate from this University. She has worked as a Professor and Principal of Government College of Engineering, Salem, Tamilnadu and has a teaching experience of 38 years. She has published more than 400 research journals in national and international conferences and journals. Her fields of Research: Pattern Recognition, Application of ANN for Image Processing, Network Security, Knowledge Management and Grid Computing. She is a fellow member of the Institute of Electrical and Electronics Engineers (IEEE), Institution of Engineering (IE), Indian Society for Technical Education (ISTE), The Institution of Electronics and Telecommunication Engineers (IETE). She also serves as Board of member in various colleges/universities in framing curriculum and syllabus. She has supervised more than 180 postgraduate and $70 \mathrm{Ph} . \mathrm{D}$ research scholars in the field of computer science, Information and Communication. She is also served as a board member for several International and national journals. 